\title{
Movimiento corporal y aprendizaje del inglés: Significados afectivos de los infantes
}

\author{
Body movement and English language learning: Children's \\ affective meanings
}

DRA. YASNA YILORM BARRIENTOS

Universidad Austral de Chile, Valdivia, Chile (yyilorm@uach.cl)(https://orcid.org/0000-00016168-1207)

DRA. ELIZABETH MARTÍNEZ PALMA.

Universidad Austral de Chile, Valdivia, Chile (elizabethmartinez@uach.cl)(https://orcid org/0000-0003-3960-6870)

BELÉN SILVA DOMÍNGUEZ

Colegio Gandhi, Valdivia, Chile (millaraay@gmail.com)(https://orcid.org/0000-0002-9611-2618) NATALIA ALARCÓN ALARCÓN

Escuela de Niebla Juan Bosch, Valdivia, Chile (nati.alarcon08@gmail.com)(https://orcid org/0000-0001-8519-9837)

\section{RESUMEN}

El presente estudio se propone explorar los significados afectivos de las niñas y los niños de escuelas valdivianas en Chile acerca de la implementación de actividades que incluyen el movimiento corporal en el aula de inglés. Las investigaciones en el área han evidenciado que el movimiento corporal tiene un impacto significativo en los procesos de enseñanza aprendizaje, permitiendo el desarrollo psicofisiológico de los infantes, mediante la producción de hormonas del disfrute y bienestar, los cuales conducen a niveles más alto de concentración, percepción, comprensión y construcción de los conocimientos. En este contexto, la inclusión del movimiento corporal en el aula de inglés, particularmente en la implementación de juegos y técnicas teatrales, representa una estrategia didáctica viable y efectiva para el desarrollo de las habilidades comunicativas en la lengua extranjera, por cuanto promueve la motivación integrativa y reduce notoriamente los altos niveles de ansiedad y de estrés, producidos por la complejidad de estar expuesto a una lengua extranjera. La metodología de investigación está enmarcada en el paradigma cualitativo y se lleva a cabo a través de un estudio descriptivo de una muestra de 50 escolares, entre 7 y 10 años, de escuelas públicas chilenas. La discusión de los resultados evidencia que estos participantes demuestran altos niveles de satisfacción ante las actividades en la que se incorpora el movimiento corporal 
para el aprendizaje del inglés destacando la apropiación, comprensión de nuevos mensajes y producción de palabras, en un ambiente escolar creativo, amistoso y entretenido.

\section{ABSTRACT}

This study purports to explore public school Chilean children's affective meanings in relation to the implementation of body movement-based activities in English classrooms from Valdivia city. Research has shown that body movement has a meaningful impact on teaching learning processes. Children's physiopsychological development is strengthened through enjoyment hormone production and wellbeing, which produces higher levels of concentration, perception, understanding and knowledge construction. The incorporation of body movement in the English classroom, particularly the implementation of games and drama techniques, stands for a viable and effective didactic strategy; integrative motivation increases and high levels of anxiety and stress, caused by the complexity of being exposed to a foreign language, are notoriously reduced. Therefore, communicative skills in the foreign language can be successfully developed. This research, which uses a qualitative research method, is carried out through a descriptive study, whose sample is composed of fifty 7 to 10-yearolds from Chilean public schools. The result discussion shows that participants are highly satisfied with activities that incorporate body movement for the English learning process and highlight the appropriation, understanding of new messages and production of words in a creative, friendly and amusing school environment.

\section{PALABRAS CLAVES / KEY WORDS}

Aprendizaje del inglés, infantes, movimiento corporal, motivación, significados afectivos / English learning, children, body movement, motivation, affective meanings

\section{INTRODUCCIÓN}

Los resultados de la investigación científica han evidenciado el impacto del movimiento corporal en el desarrollo afectivo y la experiencia de aprendizaje vivenciada por niñas y niños (Wells, 2012; Backes, et al., 2015; Grieco et al., 2016; Shoval et al., 2018; Toro y Sabogal, 2018). La acción de moverse no solo responde a la naturaleza del ser humano en su adaptación al mundo, sino también fortalece el procesamiento de la información, facilitando particularmente la comprensión de nuevos mensajes y la emisión de palabras dentro del acto comunicativo. La base de este proceso psicofisiológico se encuentra en el desarrollo motivacional y los estados de goce y disfrute que produce el movimiento 
corporal. La articulación de neurotransmisores libera hormonas, tales como la dopamina, a través de la corriente sanguínea que producen satisfacción y estados anímicos positivos, lo que se traduce en bienestar personal y social favoreciendo, de este modo, los procesos de enseñanza aprendizaje, por cuanto la niña y el niño manifiestan mejor disposición cognitiva y afectiva para la construcción de conocimientos y cultura (Erickson, et al., 2015; Benes et al., 2016; Martin y Murtagh, 2017). Al realizar este tipo de actividad, se evidencia un incremento del flujo sanguíneo que se traduce en energía, reducción de estrés, y producción hormonal favoreciendo la conexión entre las células cerebrales (Caspersen, Powell, \& Christenson, 1985; Erlauer, 2003).

En lo que respecta el aprendizaje del inglés como lengua extranjera, la incorporación del movimiento corporal en actividades lúdicas y de dramatización durante las sesiones de clase, se visualiza como una estrategia didáctica que revoluciona los paradigmas tradicionales; por cuanto están centradas en la motivación integrativa (Richard-Amato, 2009; Krashen, 2012) y en la construcción de espacios educativos acogedores que disminuyen los niveles de ansiedad provocados por el encuentro con una lengua que es diferente a la materna. De igual modo, cabe mencionar que estas estrategias aportan al desarrollo de las habilidades comunicativas que son necesarias para decodificar y codificar mensajes en una lengua extranjera. Participar en actividades lúdicas y juegos de roles da apertura a la interacción, al diálogo, al despliegue de la imaginación y al trabajo colaborativo priorizando el aprendizaje significativo.

El objetivo de este artículo es explorar los significados afectivos de las niñas y los niños de escuelas valdivianas en Chile acerca de la implementación de actividades que incluyen el movimiento corporal en el aula de inglés. La metodología de investigación está enmarcada en el paradigma cualitativo y se lleva a cabo a través de un estudio descriptivo de escolares de escuelas públicas chilenas.

Los resultados de este estudio se proponen contribuir a la implementación de prácticas pedagógicas centradas en la y el estudiante, haciendo hincapié a situar estas propuestas metodológicas en la pedagogía local. La incorporación del movimiento corporal resulta necesaria para generar nuevos espacios comunicativos que consideren el rol del juego, la afectividad, la motivación y el bienestar personal y grupal como factores protectores para el desarrollo integral de los infantes.

\section{MARCO TEÓRICO}

\section{MOVIMIENTO CORPORAL Y APRENDIZAJE DE LA LENGUA INGLESA}

La naturaleza del ser humano lo conlleva a mantenerse en constante movimiento y en cada acción que lleva a cabo, establece un compromiso con su ser. "[C]ada acción posee un carácter simbólico que surge de su subjetividad y de la relación con otros" (Toro y Sabogal, 2018, p.33). 'Bajo estas circunstancias, resulta perjudicial para el individuo, desde el punto de vista físico y mental, permanecer quieto y concentrarse durante extensos periodos de tiempo (Wells, 2012). En el ámbito educativo en particular, la inclusión del movimiento corporal en el aula impacta significativamente los procesos de enseñanza-aprendizaje,

1 [1] La traducción fue hecha por las autoras. 
permitiendo que las y los niños obtengan mayores logros académicos (Shoval, et al., 2018, p.33).[1] Por su parte, Backes, et al. (2015) afirman que el movimiento corporal es determinante para que los infantes logren mayores niveles de concentración, percepción del mundo, comprensión del conocimiento, y adquisición de la consciencia como parte de un contexto, bases fundamentales del aprendizaje significativo. El movimiento constituye una forma de simbolizar, crear, entender y expresar significados, lo cual revela que la comprensión y aplicación del movimiento corporal en la sala de clase pueden ser beneficiosos para el desarrollo cognitivo y afectivo de los escolares. Existe, por ende, "una conexión positiva entre movimiento y aprendizaje" (Wells, 2012, p.1).

La actividad física, considerada como un tipo de movimiento corporal, cumple un rol fundamental en la vida de las niñas, los niños y sus profesores, especialmente en los primeros años de escolarización (Grieco et al., 2016). Presenta diversas ventajas, tales como la participación activa de los miembros del proceso de enseñanza-aprendizaje, aunque se trate de una actividad breve y con intensidad moderada. Ésta permite desarrollar la plasticidad natural del cerebro, lo cual mejora su salud y el rendimiento cognitivo (Erickson, et al., 2015; Benes et al., 2016; Martin y Murtagh, 2017). Al realizar este tipo de actividad, se evidencia un incremento del flujo sanguíneo que se traduce en energía, reducción de estrés, y producción hormonal favoreciendo la conexión entre las células cerebrales (Caspersen, et al., 1985; Erlauer, 2003). Es un hecho que el sedentarismo de las y los estudiantes durante un periodo determinado de tiempo puede causar una disminución de los niveles de glucosa y oxígeno en el cerebro provocando un déficit en las capacidades de focalización, comprensión y recuerdo (Madigan, 2004, como se citó en Reilly et al., 2012, p. 63). Estas capacidades son, por ende, fundamentales para procesar información a nivel cognitivo, fortalecer la memoria procedimental y consolidar el aprendizaje significativo.

Es necesario precisar que en las vivencias de la niña y del niño, el movimiento corporal es intrínseco debido a que su forma de interacción con el mundo requiere que “[...] se ponga de pie, a caballo, se siente, pruebe, coma, se mueva, juegue, construya o corra. Esto crea una fuente mayor, más compleja, y en conjunto más amplia de información sensorial al cerebro que la simple actividad cognitiva" (Jensen, 2010, p. 152). El aprendizaje corporal permite recordar las acciones, respuestas o conductas de manera clara, positiva y a largo plazo (Jensen, 2010). Cabe mencionar que, según Reilly et al. (2012) y Willis (2008), resulta de vital importancia realizar, en el contexto académico, cualquier tipo de actividad física, con el fin de aumentar los neurotransmisores, particularmente la dopamina, la cual es liberada durante vivencias placenteras y mejorar, así, los niveles de concentración y memoria, y fortalecer la capacidad de disfrute durante los procesos educativos en el aula. Finalmente, es posible destacar que las actividades didácticas que incorporan movimientos corporales contribuyen al desarrollo de la formación integral de las y los estudiantes (Trost, 2009; Grieco, et al., 2016; Bartholomew et al., 2018; Dinkel, et al., 2017).

En el contexto educativo chileno, las bases curriculares propuestas por el Ministerio de Educación, promueven la incorporación del movimiento corporal en los espacios educativos de manera transversal, es decir, todas las asignaturas del plan de estudio incentivan la implementación de actividades que permiten que las y los estudiantes tengan la posibilidad de moverse en el aula de manera activa (MINEDUC, 2022a). En lo que respecta particularmente al aprendizaje del inglés como lengua extranjera, es posible afirmar que el movimiento corporal de los escolares es una estrategia didáctica alternativa de enseñanzaaprendizaje de lenguas, que transforma la instrucción tradicional en un proceso educativo a 
disposición de las necesidades e intereses de las y los estudiantes (Mulatu, y Bezabih, 2018; Cabrera-Solano et al., 2020). De este modo, las habilidades comunicativas que las niñas y los niños desarrollan en el aula de inglés permiten un crecimiento significativo en el ámbito intelectual y personal, debido a que existe la posibilidad de familiarizarse con múltiples culturas, tradiciones y formas de pensar y sentir (MINEDUC, 2022b). En este contexto, como ya se ha mencionado anteriormente, se comprende que aprende la lengua inglesa trasciende el desarrollo cognitivo de los infantes, permitiendo, de manera equitativa, su desarrollo socioafectivo y comunicativo (Yilorm y Martinez, 2022).

\section{DESARROLLO SOCIO-AFECTIVO Y MOVIMIENTO CORPORAL COMO ESTRATEGIAS DIDÁCTICAS}

Para aprender una lengua extranjera, es decir, un medio de comunicación que no es el que utilizamos diariamente en nuestras situaciones sociales de desarrollo, la creación de un ambiente escolar amistoso y acogedor es determinante para facilitar los procesos educativos. La reducción del nivel de la ansiedad y de estrés sostiene la construcción de una sala de inglés como lengua extranjera (García, 2016) debido a que contribuye al desarrollo de la confianza en sí mismo y a la buena disposición para participar de manera activa y comprometida (Ratey, 2008, como se citó en Helgeson, 2011; Cañas y Chacón, 2015). Cabe mencionar que los estados de ánimo positivos pueden asociarse con un mejor rendimiento en el aula de lenguas extranjeras (Zabetipour \& Pishghadam, 2016). Por ello, el movimiento corporal, como una acción que la niña y el niño requieren para complementar su bienestar físico y mental, entretiene y alivia las tensiones permitiendo mayor focalización en los mensajes emitidos y dando apertura a la creación de esquemas cognitivos para la decodificación. Como lo indica Willis (2008), cuando las y los niños realizan acciones que les agradan, se sienten relajados y cómodos, y muestran interés frente a la tarea, el aprendizaje cobra sentido y efectividad.

De acuerdo con Richard-Amato (2009), el dominio afectivo incluye diversas variables que pueden beneficiar el proceso de adquisición de una segunda lengua, dependiendo si éstas son positivas o negativas, el grado en el que están presentes, y las combinaciones en las cuales se encuentran. Con el fin de explorar adecuadamente las dimensiones afectivas que actúan durante este proceso educativo, resulta necesario comprender que la motivación integrativa y los niveles de ansiedad juegan un rol crucial en su desarrollo. La motivación integrativa constituye el interés sincero y personal por aprender una lengua extranjera y el deseo de interactuar con las y los hablantes nativos y desarrollar una actitud positiva hacia ellas y ellos y sus culturas (Gardner y Lambert, 1972, citado en Hernández, 2006; Rozmatovna, 2020; Gholami, et al., 2012; Chee Hong y Ganapathy, 2017). Esta buena disposición para aprender la lengua extranjera facilita el desarrollo de las habilidades lingüísticas de manera significativa. Mientras más altos sean los niveles de motivación integrativa, mejores serán los logros académicos (Dörnyei, 2019; Hyun Kim y Won Shin, 2021); no obstante, este estado también puede verse afectado cuando existen factores socio-afectivos internos y externos que aumentan considerablemente los niveles de ansiedad de las y los estudiantes. La ansiedad, como un subsistema del temperamento puede ser reconocida como una respuesta psicofisiológica ante preocupaciones y eventos potencialmente peligrosos (Céspedes, 2002). En la clase de inglés, esta respuesta emocional negativa es reconocida como un tipo de ansiedad que se ocasiona por una situación propia del aprendizaje de la lengua extranjera. Es uno de los factores afectivos que más impacta a las y los estudiantes impidiendo, en algunas ocasiones, que se alcancen los logros académicos. En otras palabras, este tipo de 
ansiedad situacional desarrolla el miedo entre las y los aprendices (Liu, 2006; Zheng y Chen, 2018; Suciati, 2020). En este escenario, el movimiento corporal en conjunto con el juego y las técnicas teatrales, como estrategias didácticas liberadoras de estrés, permiten facilitar la motivación y mantener bajos niveles de ansiedad.

Dentro de las prácticas pedagógicas comunicativas para la adquisición de una segunda lengua, es menester hacer particular referencia a los métodos TPR (Total Physical Response) (1974) y TPRS (Total Physical Response Storytelling) (Cuentacuentos con gestos) (1990s), los cuales tienen como objetivo máximo, mediante la incorporación de la comunicación verbal y no verbal entre los profesores y estudiantes, alcanzar el desarrollo de la comunicación, pero en forma progresiva, desde lo más simple a lo más complejo respetando los ritmos, intereses y preferencias sensoriales de cada estudiante e implementando actividades acordes a su edad y contextos socio-culturales. El movimiento corporal como parte de los juegos y la actuación contribuyen a disminuir los niveles de ansiedad y a enviar mensajes que puedan ser comprendidos por los estudiantes mediante la gestualidad y/o el empleo del material didáctico multisensorial. Al implementar estos métodos, [I]a acción y el habla se combinan en un clima informal y libre de estrés, facilitando, a través de los gestos, la comprensión de los mensajes emitidos en la lengua adicional. "Los educandos actúan con sus cuerpos, así como también con sus mentes - en otras palabras, con su ser integral (Richard-Amato, 2009, p.158). Su principal atractivo es que, al escuchar instrucciones, historias familiares, interesantes y que responden a sus necesidades, los estudiantes se motivan con facilidad para utilizar la expresión corporal con el fin de dramatizar las historias que van oyendo y de esta manera, decodificar los mensajes oídos (Curtain, y Dalhberg, 2010; Ray y Seely, 2008). En ambos casos, se promueve el principio pedagógico que tiene relación con practicar, con mayor dedicación, la habilidad de comprensión auditiva, previo a que los estudiantes puedan expresarse verbalmente (Yilorm, et al., 2019).

Las niñas y los niños son aprendices activos, poseen un alto nivel de motivación, especialmente por la innovación, se comprometen con sus quehaceres y desafíos de manera natural y fluida, y se adaptan, con alto interés, a la diversidad de vivencias que les brinda el ambiente mediante sus sentidos. Por tanto, las tareas asignadas deben contemplar el movimiento, la actuación y los juegos. En este escenario, las profesoras y los profesores de inglés requieren diseñar estrategias didácticas que consideren la manera en que los infantes adquieren conocimientos y le otorgan significado al mundo que les rodea, como, por ejemplo, cuentos, diálogos y juegos. En esta experiencia de interacción, el uso de la lengua extranjera cumple un rol fundamental para que las niñas y los niños participen de actividades que estimulen el movimiento corporal (Brown, 2007, citado en MINEDUC, 2022). Como ya se ha mencionado anteriormente, estas estrategias facilitan en las y los estudiantes el desarrollo de habilidades para relacionar, codificar y construir nueva información que se comparte en la sala de clase; ya que estimulan la creación de más redes neuronales, las cuales conducen a mejores probabilidades para recuperar fácilmente la información (Zadina, 2008, citado en Cañas y Chacón, 2015). En lo que respecta a la lengua inglesa, esta activación neuronal permite que se complete el circuito dialógico del proceso de aprendizaje de la lengua extranjera: A mayor nivel de motivación, mayor es la apertura a los mensajes emitidos por otros, mayor es la comprensión e internalización de los contenidos y contextos, y mayor es la capacidad de producción de nuevos mensajes en la lengua extranjera (Hastings, comunicación personal, 2007). 


\section{JUEGOS Y TÉCNICAS TEATRALES}

Las estrategias didácticas lúdicas otorgan a las niñas y a los niños oportunidades para construir significados de los territorios que habitan, desarrollar la creatividad y la imaginación dando apertura a diversas posibilidades de aproximación al conocimiento del mundo propio y comunitario, con el fin de articular la representación de sentidos de realidad para la construcción de una identidad personal y social (Yilorm y Martínez, 2022). De manera particular, los juegos y las técnicas teatrales constituyen actividades lúdicas entretenidas y desafiantes que forman parte del desarrollo natural de los infantes mejorando las relaciones interpersonales y potenciando la capacidad de relajar la mente y el cuerpo (Andreu y García, 2000; Chamorro, 2010; Gray, 2011; Ur, 1991; Yilorm \& Acosta, 2015).

Chamorro (2010) establece que los juegos desarrollan las habilidades que las niñas y los niños necesitan adquirir para expandir su creatividad, resolver problemas de manera efectiva, y mejorar el uso del lenguaje y los roles sociales. De este modo, no cabe duda que los juegos son útiles para potenciar la motivación de los aprendices y recrear contextos similares a la vida real, y por tanto, altamente significativos para recordar mejor la lengua adicional (Stakanova y Tolstikhina, 2014). Junto con los juegos, las técnicas teatrales también son estrategias didácticas efectivas y óptimas para la inclusión del movimiento corporal. La actuación, como técnica de enseñanza, es "un proceso social activo que desarrolla la capacidad de la niña y del niño para los juegos de roles-para proyectarse en roles imaginarios, personajes y situaciones-como una forma de explorar y expresar ideas a través del cuerpo y de la voz" (McGregor, et al., 1977, p. 4). Al implementar técnicas teatrales, las y los docentes se focalizan no solo en la lengua extranjera sino también en el cómo la lengua puede ser utilizada y expresada de diferentes maneras y en diferentes contextos, a través de los cuales el cuerpo y la imaginación de las niñas y de los niños son los canales para que esto ocurra (Zyoud, 2010; Alcedo et al. 2011). En resumen, se evidencia que, con el fin de consolidar un aprendizaje significativo en el aula de inglés, las actividades didácticas deben proponerse no sólo el desarrollo de las habilidades lingüísticas y comunicativas de los escolares, sino también, elevar sus niveles de motivación en un ambiente que propicie su bienestar. Tal como lo menciona Barreira (2018), las actividades tales como el juego, la música, las representaciones, el movimiento corporal y la manipulación son idóneas porque responden a sus intereses y necesidades.

\section{METODOLOGÍA}

Este trabajo está enmarcado en el paradigma cualitativo y se lleva a cabo mediante un estudio de carácter descriptivo de una muestra de 50 niñas y niños de entre 7 y 10 años de escuelas municipales de la ciudad de Valdivia, Chile. El estudio explora los significados afectivos de escolares en relación a las experiencias de aprendizaje desarrolladas durante la clase de inglés y basadas en el movimiento corporal, el juego y las técnicas teatrales. El método de recolección de información es la entrevista semi-estructurada con preguntas abiertas diseñadas para las y los estudiantes que participaron del estudio (Cohen y Crabtree, 2006; Merriam y Tisdell, 2016; Creswell y Creswell, 2018). La población contempla un total de 26 instituciones educativas de la ciudad de Valdivia, las cuales son parte del proyecto Let's go for English in 100 fun ways (2015). De las 26 escuelas, este artículo considera un total de 
15 establecimientos públicos. La muestra, cuya selección fue la disposición para participar de las instancias de la investigación, está constituida por 50 infantes que pertenecen a los 15 colegios intervenidos. Esta muestra considera las producciones orales de los escolares como textos de análisis.

El método de investigación utilizado consiste en el análisis de contenido, definido como una herramienta que procesa información para identificar, seleccionar, organizar, analizar e informar, de manera detallada, patrones o campos temáticos que se infieren e interpretan en la lectura y relectura minuciosa de la información recogida, para la obtención de resultados del estudio (Braun \& Clarke, 2006; Mieles, et al., 2012).

Para el análisis de los resultados se han considerado las categorías emergentes que se identifican a partir de la selección de actividades pedagógicas implementadas en aulas escolares de inglés en instituciones educativas municipales de la ciudad de Valdivia, Chile. Este procedimiento constituye la primera etapa del análisis de la información recopilada para el estudio, la cual clasifica las experiencias de aula (actividades pedagógicas), que involucran el movimiento, como aspecto central del aprendizaje de la lengua inglesa. El proceso selectivo se lleva a cabo a partir de la pregunta de investigación que guía el diseño metodológico. De igual modo, se incluyen los marcos conceptuales sobre movimiento corporal, la actividad física, estrategias lúdicas y técnicas teatrales, para construir las bases teóricas de las categorías inferidas, las cuales se describen a continuación:

- Asociación movimiento corporal, gestualidad e interiorización de conceptos y proposiciones: En esta categoría, el aprendizaje corporal es un indicador que permite evidenciar, en las expresiones orales de niñas y niños, la relación entre movimiento corporal y apropiación léxico gramatical y semántica de la lengua inglesa. Según los referentes teóricos, el movimiento constituye una forma de simbolizar, crear, entender y expresar significados, los cuales hacen referencia, en el caso de este estudio, a las apreciaciones que emiten los infantes respecto a las actividades vivenciadas desde una perspectiva afectiva, y la justificación que utilizan para argumentar tales valoraciones.

- Movimiento Corporal para el desarrollo de actividades pedagógicas: Esta categoría considera que el aprendizaje corporal facilita el desarrollo de habilidades de relación, codificación y estructuración de nueva información, debido a que estimula la creación de redes neuronales que mejoran las funciones de procesamiento de la memoria, lo cual permite recordar acciones, respuestas o conductas a largo plazo (Jensen, 2010). De igual modo, la actividad física aumenta el flujo de neurotransmisores que favorecen la capacidad de disfrute durante las vivencias placenteras, las cuales impactan en la motivación de quienes participan de ellas. Desde esta perspectiva, en esta categoría se identifican las valoraciones afectivas de los infantes, codificadas en sus producciones orales, respecto al proceso vivenciado en las actividades pedagógicas que involucran el movimiento corporal mediante el juego.

- Representación y dramatización corporal: Esta categoría comprende la dramatización corporal, como espacios de acción en que las niñas y los niños pueden representar personajes y situaciones, vivenciar juegos de roles y expresarse verbal y corporalmente experimentando con diferentes códigos, a través de la imaginación y la creatividad. Para el caso de esta unidad de análisis, se evidenciaron las evaluaciones emitidas por los participantes con respecto a su experimentación en las actividades pedagógicas 
implementadas, las cuales consideran el uso de técnicas teatrales.

Las tres categorías expuestas se manifiestan en actividades que incorporan la acción lúdica como mecanismo potenciador del aprendizaje. De este modo, se ha considerado la inclusión del juego como proceso que estimula el lenguaje, la memoria, el razonamiento, la imaginación, la creatividad, etc. Así mismo, desde una perspectiva más social, la dramatización contribuye al desarrollo de habilidades socio-cognitivas, comunicativas y afectivas (Rodrigo, 2014).

En una segunda etapa, se analizan las expresiones emitidas por los infantes respecto a los significados afectivos que derivan de su participación en las actividades pedagógicas seleccionadas (implementadas en las clases de inglés), relacionándolas con la justificación de sus valoraciones, manifiestas en estos significados, según el proceso vivido. El análisis de estas producciones orales se lleva a cabo a partir de las categorías emergentes presentadas anteriormente.

En la tercera etapa de análisis, se identifican las relaciones de sentido que se construyen entre categorías y producciones orales infantiles. En este nivel se pretende evidenciar el posicionamiento de niñas y niños con respecto al desarrollo e implicación personal en sus aprendizajes, durante actividades que involucran movimiento corporal en el ámbito de la lengua inglesa.

\section{ANÁLISIS Y DISCUSIÓN DE LOS RESULTADOS}

De acuerdo con las categorías de análisis evidenciadas en el estudio, es posible advertir la actividad placentera y de disfrute que genera en las niñas y niños la posibilidad de incorporar el movimiento corporal en los espacios educativos en donde se aprende la lengua inglesa. En este caso, el movimiento corporal y la gestualidad, como mecanismo de organización de esquemas conceptuales, el movimiento en diversas dimensiones y la dramatización corporal, han sido significativamente valorados por los infantes participantes de la investigación, debido a su implicancia en sus aprendizajes.

\section{Cuadro 1: Síntesis de las producciones orales emitidas por los participantes del estudio}

\section{Asociación movimiento corpo- \\ ral, gestualidad e interiorización de conceptos y proposiciones}

Wind up your robot!

“Sí, me gustó porque aprendí algunas palabras."

Wind up your robot!

"Me gustó porque fue muy entretenida."
Movimiento Corporal para el desarrollo de actividades pedagógicas

Hide and Seek

"Sí se aprende jugando, si me gustó."

Hide and Seek

“A mí me gustó la escondida y mi opinión es si."
Representación y dramatización corporal

Let's Go Fishing

"Muy divertido porque es fácil."

Let's Go Fishing

"Es una manera divertida de aprender inglés." 
Cuadro 1: Síntesis de las producciones orales emitidas por los participantes del estudio

\begin{tabular}{l|l|l|l}
$\begin{array}{c}\text { Asociación movimiento corpo- } \\
\text { ral, gestualidad e interiorización } \\
\text { de conceptos y proposiciones }\end{array}$ & $\begin{array}{c}\text { Movimiento Corporal para el } \\
\text { desarrollo de actividades peda- } \\
\text { gógicas }\end{array}$ & $\begin{array}{l}\text { Representación y dramatización } \\
\text { corporal }\end{array}$ \\
$\begin{array}{l}\text { Rock, Paper, scissors } \\
\text { "A mí me gustó el cachipún por- } \\
\text { que es entretenido al igual que en } \\
\text { inglés. Es divertido." }\end{array}$ & $\begin{array}{l}7 \text { up } 2 \\
\text { "Estuvo muy entretenido y es una } \\
\text { bonita forma de aprender y refor- } \\
\text { zar lo que sabemos." }\end{array}$ & $\begin{array}{l}\text { Shh, be quiet } \\
\text { "Yo opino que la Minnie es muy } \\
\text { floja pero nos ayuda a estar calla- } \\
\text { dos." }\end{array}$ \\
\hline $\begin{array}{l}\text { Rock, Paper, scissors } \\
\text { "Si, me gustó mucho y aprendí a a } \\
\text { decir varias cosas más." }\end{array}$ & $\begin{array}{l}\text { Go for a seat! 2 } \\
\text { "Me gustó mucho y aprendí mu- } \\
\text { cho." }\end{array}$ & $\begin{array}{l}\text { Shh, be quiet } \\
\text { "A mí me gustó trabajar con la } \\
\text { Minnie porque ella siempre esta- } \\
\text { ba dormida y nos hacía callar." }\end{array}$ \\
\hline & $\begin{array}{l}\text { Go for a seat! 2 } \\
\text { "Me gustó mucho porque era muy } \\
\text { entretenido" }\end{array}$ & \\
\hline
\end{tabular}

Significados afectivos expresados en las producciones orales infantiles según categoría de análisis

En el caso de la categoría,"Asociación movimiento corporal, gestualidad e interiorización de conceptos y proposiciones", las actividades seleccionadas, Wind up your robot! y Rock, paper, scissors, hacen referencia al movimiento corporal que se manifiesta como una respuesta inmediata al mensaje oído en la lengua extranjera. En el primer caso, las y los estudiantes juegan a convertirse en robots. Escuchan instrucciones simples y se mueven por el aula, convirtiendo los mensajes en acciones, tales como caminar hacia atrás, saltar como un canguro y/o dibujar en la pizarra y saltar al mismo tiempo. En el segundo ejemplo, las y los estudiantes interactúan mediante una actividad lúdica conocida en su entorno; se asocian experiencias de vida de manera positiva entre ambas culturas solicitando que los escolares emitan palabras en la lengua extranjera: Rock, paper, scissors, y ejecuten una acción que represente uno de los tres conceptos simultáneamente.

En las expresiones de los participantes del estudio se evidencia una apropiación del espacio-tiempo que se construye a través de la actividad de aprendizaje desde el gusto y satisfacción. Como se aprecia en los siguientes ejemplos correspondientes a: Wind up your robot! “Sí, me gustó porque aprendí algunas palabras”, “Me gustó porque fue muy entretenida.", la justificación del espacio placentero, que brinda el desarrollo de la actividad, se centra en dos aspectos fundamentales. El primero de ellos guarda relación con el aprendizaje como centro operador para internalizar nuevos mensajes los cuales se asocian, en el plano comunicativo, al reconocimiento y apropiación de "palabras", en este caso en otra lengua. El segundo aspecto, hace referencia al disfrute que se experimenta durante el desarrollo de la tarea por su nivel de entretención, pues la dinámica movimiento y apropiación de nuevos conceptos resulta ser un espacio de gusto y disfrute.

Los campos temáticos que justifican el gusto por lo vivenciado en Rock, paper, scissors se pueden observar, del mismo modo que la actividad anterior, en los siguientes ejemplos, "A mí me gustó el cachipún porque es entretenido al igual que en inglés. Es divertido", "Si, me gustó mucho y aprendí a decir varias cosas más". En estos casos, el juego cumple un 
rol fundamental en la valoración que emiten los infantes respecto a su participación en la experiencia de aprendizaje, pues los significados afectivos que se construyen respecto de la satisfacción percibida, por lo entretenido y divertido de la actividad, son asociados representacionalmente a la lengua inglesa. En el segundo ejemplo, el gusto se relaciona con el aprendizaje del inglés a partir de habilidades de comprensión y producción de la lengua "aprendí a decir varias cosas más", pues se amplía en campo de posibilidades de emisión de nuevos conceptos, y se posiciona una autoevaluación consciente y positiva del logro obtenido, lo que puede incidir en la autovaloración y autoestima del infante. La relación entre la conciencia del aprendizaje obtenido y la percepción de una instancia agradable otorgada por la actividad, pueden ser factores que facilitan la motivación y reorganizan las respuestas ansiosas que pudieran provocarse al decodificar mensajes en una lengua distinta a la materna.

En conclusión, es posible advertir que los significados afectivos de gusto y disfrute que se construyen a partir de esta categoría, en las producciones orales de los infantes, son justificados por aspectos claves tales como: el aprendizaje, la entretención y la diversión, los cuales son asociados a la organización cognitiva de nuevos conceptos en la lengua inglesa. Estos aspectos se centran, de manera particular, en el reconocimiento de las habilidades que desarrolla la niña y el niño, y la valoración de su proceso de aprendizaje.

Sobre la base de la categoría,"Movimiento Corporal para el desarrollo de actividades pedagógicas", las experiencias seleccionadas Hide and seek, 7 up y Go for a seat! tienen como objetivo que los estudiantes permanezcan activos durante la sesión de clase y adquieran los conocimientos y la cultura de manera significativa. Basándose en sus propias experiencias culturales, el juego como el de la escondida y la de intercambiar rapidamente de asiento para no quedarse de pie, les permitirá vincularse con el movimiento, la alegría, el suspenso y la sorpresa.

De acuerdo con las expresiones que manifiestan las niñas y los niños luego de compartir de estas experiencias de aprendizaje, las acciones de juego y la percepción valorativa de las mismas (entretención, actividad bonita), son aspectos que se combinan, como ejes articuladores, en el gusto que significa afectivamente sus estados emocionales frente a la tarea ejecutada y posiciona sus aprendizajes; como se observa en los siguientes ejemplos: "Sí se aprende jugando, si me gustó", “A mí me gustó la escondida y mi opinión es si”, "Me gustó mucho y aprendí mucho" y “Me gustó mucho porque era muy entretenido".

En esta categoría, se incorpora un segundo aspecto que refuerza la valoración afectiva y la percepción del aprendizaje como un espacio de reconstrucción del conocimiento. En este caso, se conjugan entretención, aprendizaje y reforzamiento del saber cómo un todo organizador que otorga significados a la experiencia y orienta la motivación integrativa a nivel colectivo: "Estuvo muy entretenido y es una bonita forma de aprender y reforzar lo que sabemos". Por tanto, no se individualiza el saber sino que se proyecta de manera colaborativa.

En definitiva, en esta categoría, la relación juego y movimiento posicionan el estado emocional y afectivo de los estudiantes generando motivación, bienestar y seguridad para el aprendizaje del inglés como lengua extranjera, lo que facilita la apropiación de nuevos mensajes. Estos estados emocionales disminuyen los niveles de ansiedad que pueden ser generados por el estrés y la falta de acceso a los nuevos códigos lingüísticos a los que se enfrentan los escolares. 
Para la tercera categoría expuesta en este análisis, “Representación y dramatización corporal", el juego de roles, la representación de personajes y la actividad compartida a través del movimiento y la gestualidad, se constituyen aspectos claves para el reconocimiento personal y el desarrollo de relaciones sociales. En este caso, las actividades Let's go fishing! y Shh, be quiet! sitúan el aprendizaje a partir de acciones en las que las y los estudiantes deberán asumir nuevos roles relacionados con la vida real: la pesca y los rituales que se experimentan en los hogares cuando una niña y/o un niño se duerme. A través de estas técnicas teatrales, los infantes se focalizarán en sus nuevos personajes y tareas, percibirán los mensajes en la lengua extranjera desde esa perspectiva imaginaria, creativa y motivadora, y decodificarán los mensajes con mayor naturalidad, facilitando, de este modo, el desarrollo de habilidades comunicativas y socioafectivas.

En las opiniones de las niñas y los niños, es posible advertir el alto nivel de motivación que expresan los infantes cuando participan de las experiencias pedagógicas referidas en este estudio. Este posicionamiento motivacional permite que el estudiante sea un actor protagónico del proceso comunicativo y perciba su aprendizaje como un acceso a nuevo conocimiento. La accesibilidad al conocimiento se evidencia en la facilidad y la diversión, expresada por las y los niños, que generan las actividades, como se aprecia en los siguientes ejemplos: Muy divertido porque es fácil.", Es una manera divertida de aprender inglés".

En segundo lugar, el uso del objeto personificado durante el proceso de mediación de la actividad de aprendizaje, como espacio de organización de roles sociales, facilita la simbolización de la actividad rutinaria y da apertura a la autorregulación de la conducta de las niñas y niños frente a situaciones socialmente definidas. En este ejemplo, la Minnie, en la actividad “Shh, be quiet!", representa el espacio de silencio y concentración, lo cual facilita el trabajo individual y la disposición grupal para el aprendizaje, como se distingue en las siguientes expresiones: "Yo opino que la Minnie es muy floja pero nos ayuda a estar callados", "A mí me gusto trabajar con la Minnie porque ella siempre estaba dormida y nos hacía callar." En este último caso, el gusto por el trabajo a realizar se justifica en la personificación de un objeto que, al estar dormido, genera una respuesta conductual en los infantes, la cual se asocia al silencio requerido para el ejercicio. Por tanto, de acuerdo con las edades de las niñas y niños, se identifica una evidente transición representacional que se sitúa desde la asociación de las rutinas con un objeto, hacia la simbolización de las mismas en un accionar conductual autónomo.

En definitiva, para esta categoría, la representación de roles, la dramatización y el movimiento facilitan la internalización de códigos socio-culturales que organizan la conducta y dirigen el aprendizaje. De este modo, se fortalece la autonomía y se potencia el estado de disfrute y bienestar de los infantes. 


\begin{tabular}{|c|c|c|}
\hline \multicolumn{3}{|c|}{ Cuadro 2: Tabla de resumen de resultados por categoría } \\
\hline Categoria de Análisis & $\begin{array}{l}\text { Campos temáticos abordados } \\
\text { en las producciones orales } \\
\text { infantiles }\end{array}$ & $\begin{array}{l}\text { Significados afectivos codifica- } \\
\text { dos en las expresiones de los } \\
\text { infantes }\end{array}$ \\
\hline $\begin{array}{l}\text { Asociación movimiento corporal, } \\
\text { gestualidad e interiorización de } \\
\text { conceptos y proposiciones }\end{array}$ & $\begin{array}{l}\text { Relación entre aprendizaje, entre- } \\
\text { tención y diversión }\end{array}$ & $\begin{array}{l}\text { Significados afectivos de gusto } \\
\text { y disfrute, son asociados a la or- } \\
\text { ganización cognitiva de nuevos } \\
\text { conceptos en la lengua inglesa. } \\
\text { Se percibe en los infantes, el re- } \\
\text { conocimiento de sus habilidades } \\
\text { y la valoración de sus procesos de } \\
\text { aprendizaje. }\end{array}$ \\
\hline $\begin{array}{l}\text { Movimiento Corporal para el desa- } \\
\text { rrollo de actividades pedagógicas }\end{array}$ & $\begin{array}{l}\text { Relación juego y movimiento para } \\
\text { la motivación y el bienestar }\end{array}$ & $\begin{array}{l}\text { Esta relación posiciona el esta- } \\
\text { do emocional y afectivo de los } \\
\text { estudiantes generando motiva- } \\
\text { ción, bienestar y seguridad para } \\
\text { el aprendizaje de nuevos códigos } \\
\text { lingüísticos en inglés }\end{array}$ \\
\hline $\begin{array}{l}\text { Representación y dramatización } \\
\text { corporal }\end{array}$ & $\begin{array}{l}\text { Internalización de códigos so- } \\
\text { cio-culturales que organizan la } \\
\text { conducta y dirigen el aprendizaje }\end{array}$ & $\begin{array}{l}\text { Mediante la representación, la } \\
\text { dramatización y el movimiento, se } \\
\text { fortalece la autonomía y se poten- } \\
\text { cia el estado de disfrute y bienes- } \\
\text { tar de los infantes. }\end{array}$ \\
\hline
\end{tabular}

\section{CONCLUSIONES}

En el análisis de las producciones orales emitidas por los participantes de la investigación, y las relaciones de sentido evidenciadas entre categorías, es posible advertir cómo los significados valorativos que construyen estas expresiones verbales, dan cuenta de factores que asocian el juego y el movimiento corporal como principios centrales para el aprendizaje significativo. En este contexto, el acceso a la lengua inglesa fortalece la autovaloración y la conciencia del saber construido, a través de la comprensión, la memoria y el refuerzo para procesar información adecuadamente. De igual modo, dinamiza las motivaciones intrínsecas e integrativas y posiciona afectivamente estados emocionales de bienestar y satisfacción afectiva. Estos significados se organizan en diversos campos temáticos, los cuales permiten reconocer las valoraciones emitidas por las niñas y los niños. Estos se resumen a continuación:

- La diversión y la entretención son aspectos básicos que posicionan la motivación, la satisfacción emocional y facilitan el aprendizaje del inglés como lengua extranjera.

- El movimiento corporal en coordinación con la actividad lúdica, expresadas en el juego, son factores que mediatizan el acceso a la comprensión, interiorización y decodificación de información como ejes articuladores para el aprendizaje del inglés como lengua extranjera. 
- El aprendizaje de nuevas palabras, a través de la actividad corporal, potencia el proceso de la memoria y la elaboración de esquemas cognitivos. En este sentido, las palabras y sus significados se concretan en la actividad corporal, la cual se articula con la representación mental de los conceptos, las proposiciones, y relaciones semánticas. La acción vivenciada en el movimiento sienta las bases de representaciones más abstractas inscritas en la comprensión de mensajes y producción de nuevas palabras.

- $\quad$ El aprendizaje a través del juego forma parte del desarrollo evolutivo de las niñas y los niños. El espacio lúdico instanciado en el juego, permite construir sentidos de realidad, descubrir múltiples posibilidades para entender el mundo, e intervenir desde la construcción de una identidad en proyección.

- Las actividades pedagógicas basadas en el movimiento corporal y el juego, refuerzan la construcción de patrones y esquemas de conocimientos.

- Las actividades pedagógicas que incorporan la dramatización colaboran con el modelamiento y la organización conductual. De este modo, el tránsito desde la externalización (objeto como símbolo de regulación) hacia la interiorización de la regulación conductual, según sistema de normas, da apertura a la autorregulación emocional.

- Los significados afectivos se manifiestan desde lo placentero y motivante, lo cual permite el reconocimiento y la valoración de lo aprendido. Como se mencionó en el primer aspecto, la percepción de las niñas y los niños acerca de las experiencias pedagógicas, identificada en sus respuestas, articula la diversión, la entretención y el juego como un espacio de disfrute, en tanto aprendizaje y construcción de saberes.

- Finalmente, la opinión como espacio de participación, permite la autoconciencia de la experiencia concretizada en la actividad corporal, especialmente lúdica, la cual sustenta la valoración del espacio de interacción a través del juego y su potencial para desarrollar espacios gratos de bienestar.

De acuerdo con los resultados de este estudio, resulta imprescindible incorporar, en la práctica pedagógica del inglés como lengua extranjera, el movimiento corporal a través de estrategias lúdicas y de dramatización (técnicas teatrales) para el desarrollo multidimensional de los infantes, centrado en aprendizajes significativos.

\section{REFERENCIAS}

Alcedo, Y., y Chacón, C. (2011). El enfoque lúdico como estrategia metodológica para promover el aprendizaje del inglés en niños de educación primaria. SABER. Revista Multidisciplinaria del Consejo de Investigación de la Universidad de Oriente, 23(1),69-76. https://www.redalyc.org/articulo.oa?id=427739445011

Andreu, M., y García, M. (2000, November). Actividades lúdicas en la enseñanza de LFE: el juego didáctico. In Bordoy, M., van Hooft, A., \&, Sequeros, A. (Ed). I congreso Internacional de español para fines específicos (pp. 121-125). Secretaría General Técnica.

Backes, B., Porta, M., y de Anglat, H. (2015). El movimiento corporal en la educación infantil 
y la adquisición de saberes. Educere, 19(64), 777-790. https://dialnet.unirioja.es/servlet/ articulo?codigo $=6530221$

Barreira, E. (2018). La enseñanza del inglés en el primer ciclo de la educación infantil como práctica en escuelas y centros educativos. Propuesta de un modelo de actuación. RELAdEl. Revista Latinoamericana De Educación Infantil, 4(1), 211-243. https://revistas.usc.es/ index.php/reladei/article/view/4867

Bartholomew, J., Golaszewski, N., Jowers, E., Korinek, E., Roberts, G., Fall, A., y Vaughn, S. (2018). Active learning improves on-task behaviors in 4th grade children. Preventive medicine, 111, 49-54.

Benes, S., Finn, K., Sullivan, E., y Yan, Z. (2016). Teachers' perceptions of using movement in the classroom. The Physical Educator, 73(1), 110-135. 10.18666/TPE-2016-V73-I1-5316

Braun, V., y Clarke, V. (2006) Using thematic analysis in psychology, Qualitative research in psychology, (3)2, 77-101. http://dx.doi.org/10.1191/1478088706qp063oa

Cabrera-Solano, P., Quinonez-Beltran, A., Gonzalez-Torres, P., Ochoa-Cueva, C., y CastilloCuesta, L. (2020). Enhancing EFL students' active learning by using 'formative' on mobile devices. International Journal of Emerging Technologies in Learning (iJET), 15(13), 252263. https://www.learntechlib.org/p/217604/.

Cañas, L., y Chacón, C. (2015). Aportes de la neurociencia para el desarrollo de estrategias de enseñanza del inglés. Acción Pedagógica, (24), 52-61. https://dialnet.unirioja.es/servlet/ articulo?codigo $=6224811$

Caspersen, Powell, y Christenson, 1985. Physical activity, exercise, and physical fitness: definitions and distinctions for health-related research. Public Health Rep, 100(2), 126131.

Céspedes, A. (2002). Consideraciones generales para la integración en la escuela común de niños con dificultades afectivo-conductuales en G. Lucchini (Ed.), Niños con necesidades educativas especiales. Ediciones Universidad Católica de Chile.

Chamorro, I. L. (2010). El juego en la educación infantil y primaria. Autodidacta, 1(3), 19-37.

Chee Hong, Y., y Ganapathy, M. (2017). To investigate ESL students' instrumental and integrative motivation towards English language learning in a Chinese school in Penang: Case study. English Language Teaching 10(9), 17-35. 10.5539/elt.v10n9p1

Cohen, D., y Crabtree, B. (2006). Qualitative research guidelines project. Robert Wood Johnson Foundation.

Creswell, J., Creswell, D. (2018). Research design: qualitative, quantitative, and mixed methods approaches. SAGE.

Curtain, H., y Dahlberg, C. (2010). Languages and Children: Making the match, new languages for young learners, grades K-8. Pearson.

Dörnyei, Z. (2019). From integrative motivation to directed motivational currents: The evolution of the understanding of L2 motivation over three decades. In M. Lamb, K. Csizér, A. Henry \& S. Ryan (Eds.), Palgrave Macmillan handbook of motivation for language learning (pp. 39-69). Palgrave.

Dinkel, D., Schaffer, C., Snyder, K., \& Lee, J. M. (2017). They just need to move: Teachers' perception of classroom physical activity breaks. Teaching and Teacher Education, 63, 186-195.

Erickson, K., Hillman, C., y Kramer, A. (2015). Physical activity, brain, and cognition. Current Opinion in Behavioral Sciences, 4, 27-32. http://dx.doi.org/10.1016/j.cobeha.2015.01.005 Erlauer, L. (2003). The brain-compatible classroom: using what we know about learning to 
improve teaching. ASCD.

García, C. (2016). Principios del aprendizaje del cerebro y su repercusión en el proceso de adquisición-enseñanza de la lengua extranjera en la educación bilingüe. Encuentro: revista de investigación e innovación en la clase de idiomas, 25, 76-86.

Gardner, R., y Lambert, W. (1972). Attitudes and motivation in second language learning. Newbury House Publishers.

Gholami, R., Allahyar, N., y Rafik-Galea, S. (2012). Integrative motivation as an essential determinant of achievement: A case of EFL high school students. World Applied Sciences Journal 17(11), 1416-1424

Grieco, L., Jowers, M., Errisuriz, L., y Bartholomew, J. (2016). Physically active vs. sedentary academic lessons: A dose response study for elementary student time on task. Preventive Medicine, 89, 98-103. 10.1016/j.ypmed.2016.05.021

Gray, P. (2011). The decline of play and the rise of psychopathology in children and adolescents. American Journal of Play, 3(4), 443-463. https://www.researchgate.net/ publication/265449180_The_Decline_of_Play_and_the_Rise_of_Psychopathology_in_ Children_and_Adolescents

Helgeson, J. (2011). 4 Simple Ways to Add Movement in Daily Lessons. Kappa Delta Pi Record, 47(2), 80-84.

Hernandez, T. (2006). Integrative motivation as a predictor of success in the intermediate foreign language classroom intermediate foreign language classroom. Foreign Language Annals, 39(4). ttps://doi.org/10.1111/j.1944-9720.2006.tb02279.x

Hyun, S., y Won, H. (2021). Second language learners' self efficacy and English achievement: the meditating role of integrative motivation. English Teaching \& Learning 45(4), 1-14. 10.1007/s42321-021-00083-5

Jensen, E. (2010). Cerebro y Aprendizaje. Competencias e implicancias educativas. Narcea

Krashen, S. (2012). The Compelling (not just interesting) Input Hypothesis. The English Connection. KOTESOL, 15(3). 1.

Liu, M. (2006). Anxiety in Chinese EFL students at different proficiency levels. System, 34, 301-316

Martin, R., y Murtagh, E. (2017). Teachers' and students' perspectives of participating in the 'Active Classrooms' movement integration programme. Teaching and Teacher Education, 63, 218-230. 10.1016/j.tate.2017.01.002

McGregor, L., Tate, M., \& Robinson, K. (1977). Learning through drama. Heinemann Educational Books Ltd.

Merriam, S., y Tisdell, E. (2016). Qualitative research: A guide to design and implementation (4th ed.). Jossey Bass.

Mieles, M., Tonon, G., y Alvarado, S. Investigación cualitativa: el análisis temático para el tratamiento de la información desde el enfoque de la fenomenología social. Universitas Humanística 74, 195-225. http://www.redalyc.org/articulo.oa?id=79125420009

MINEDUC (2022a). Inglés. Currículum Nacional. https://www.curriculumnacional.cl/portal/ Educacion-General/Ingles/

MINEDUC (2022b). Organización curricular Inglés. Curriculum Nacional. https://www. curriculumnacional.cl/portal/Educacion-General/Ingles/20954:Organizacion-curricularIngles

Mulatu, M., y Bezabih, W. (2018). The Effects of Teachers' Perceptions on the implementations of active learning in EFL classrooms. Global Journal of Human-Social Science Research, 
18(6), 31-37.

Ray, B., y Seely, C. (2008). Fluency through TPR storytelling: Achieving real language acquisition in school. Blaine Ray Workshops.

Reilly, E., Buskist, C., \& Gross, M. K. (2012). Movement in the classroom: boosting brain power, fighting obesity. Kappa Delta Pi Record, 48(2), 62-66.

Richard-Amato, P. (2009). Making it Happen. Pearson ESL.

Rodrigo, M. (2014). Desarrollo intelectual y procesos cognitivos entre los 2 y los 6 años en J. Palacios, A. Marchesi, y C. Coll (Eds.), Desarrollo psicológico y educación. Alianza.

Rozmatovna, A.O. (2020). The influence of integrative motivation and instrumental motivation on learning English as a foreign Language. Journal of critical reviews (7)2. 10.31838/ jcr.07.12.164

Shoval, E., Sharir, T., Arnon, M., y Tenenbaum, G. (2018). The effect of integrating movement into the learning environment of kindergarten children on their academic achievements.

Early Childhood Education Journal, 46(3), 355-364. 10.1007/s10643-017-0870-x

Stakanova, E., y Tolstikhina, E. (2014). Different approaches to teaching English as a foreign language to young learners. Procedia-Social and Behavioral Sciences, 146, 456-460. https://doi.org/10.1016/j.sbspro.2014.08.154

Suciati, S. (2020). Speaking anxiety in EFL classroom: categories and factors. Edulingua: Jurnal Linguistiks Terapan dan Pendidikan Bahasa Inggris, 7(1), 39-45. 10.34001/edulingua. v7i1.1168

Toro, S., y Sabogal, S. (2018). Sentidos de la motricidad en Abya Yala. Rev. Tandem, 8-13. https://dialnet.unirioja.es/servlet/articulo?codigo $=6287407$

Trost, S. G. (2009). Active education: Physical education, physical activity and academic performance. Active Living Research, 1-8.

UNICEF. (2019). Para cada niño reimaginemos un mundo mejor. Informe anual de UNICEF 2019. Fondo de las Naciones Unidas para la Infancia. https://www.unicef.org/media/71156/ file/UNICEF-informe-

Ur, P. (1991). A course in language teaching: Practice and theory. Cambridge University Press. Wells, S. (2012). Moving through the curriculum: The effect of movement on student learning, behavior, and attitude. Rising Tide, 5, 1-17.

Willis, J. (2008). How your child learns best: Brain-friendly strategies you can use to ignite your child's learning and increase school success. Sourcebooks.

Yilorm, Y., y Acosta, H. (2015). Estudio descriptivo del empleo de juegos en escuelas públicas chilenas. Revista IPLAC, Publicación Latinoamericana y Caribeña de educación, 6, 97-106.

Yilorm, Y., y Martinez, E. (2022). Ludicidad y aprendizaje del inglés: Voces de niñas y niños socialmente vulnerados en Facultad de Filosofía y Educación (Ed.), Educación y Nueva constitución. Repensar lo educativo. Fondo Editorial UMCE.

Yilorm, Y., Martínez, E., Acosta, H. (2019). Desarrollo de la habilidad comprensión auditiva de la lengua inglesa en

estudiantes socialmente vulnerables. Atenas, 1(45), 69-82. http://atenas.umcc.cu/index.php/ atenas/article/view/442

Zabetipour, M., y Pishghadam, R. (2016). The impacts of open and closed Postures on EFL learners' perceptions of class activity. International Journal of Humanities and Cultural Studies, 270-279.

Zheng, Y., y Cheng, L (2018). How does anxiety influence language performance? From the perspectives of foreign language classroom anxiety and cognitive test anxiety. Lang Test 
Asia 8(13).https://doi.org/10.1186/s40468-018-0065-4

Zyoud, M. (2010). Using drama activities and techniques to foster teaching English as a foreign language: A theoretical perspective. Al Quds Open University, 1-11. https://www. researchgate.net/publication/297013590_Using_Drama_Activities_and_Techniques_to_ Foster_Teaching_English_as_a_Foreign_Language_a_Theoretical_Perspective 